\title{
A glossary for stem-cell biology
}

\author{
Austin Smith ${ }^{1}$
}

\section{Stem-cell biology is in a phase of dynamic expansion and is forming connections with a broad range of basic and applied disciplines. The field is simultaneously exposed to public and political scrutiny. A common language in the stem-cell community is an important tool for coherent exposition to these diverse audiences, not least because certain terms in the stem-cell vocabulary are used differently in other fields.}

Asymmetric division Generation of distinct fates in progeny from a single mitosis. Oriented division may position daughter cells in different microenvironments or intrinsic determinants may be segregated into only one daughter. Observed in some but not all stem cells and can occur in other types of progenitor cell.

Cancer cell of origin Precancerous cell that gives rise to a cancer stemcell. May be a mutated stem cell, or a committed progenitor thathas acquired self-renewal capacity through mutation.

Cancer-initiating cell General term that encompasses both cancer cell of origin and cancer stem cell.

Cancer stem cell Self-renewing cell responsible for sustaining a cancer and for producing differentiated progeny that form the bulk of the cancer. Cancer stem cells identified in leukaemias and certain solid tumours are critical therapeutic targets.

Cell replacement therapy Reconstitution of tissue by functional incorporation of transplanted stem-cell progeny. Distinct from 'bystander' trophic, anti-inflammatory or immunomodulatory effects of introduced cells.

Clonal analys is Investigation of properties of single cells. Essential for formal demonstration of self-renewal and potency.

Commitment Engaging in a programme leading to differentiation. For a stem cell, this means exit from self-renewal.

Embryonic stem cell Pluripotent stem-cell lines derived from early embryos before formation of the tissue germ layers.

Founder/ancestor/precursor cell General terms for cell without selfrenewal ability that contributes to tissue formation. In some cases they generate tissue stem cells.

Immortal strand The hypothesis of selective retention of parental DNA strands during asymmetric self-renewal. Potential mechanism to protect stem cells from the mutations associated with rep lication.

In vitros tem cell Self-renewal ex vivo in cells that do notovertly behave as stem cells in vivo. Occurs due to liberation from inductive commitment signals or by creation of a synthetic stem-cell state.

Label-retaining cell Candidate for adult tissue stem cell because of slow division rate and/or immortal strand retention. Interpret with caution.

Lineage priming Promiscuous expression in stem cells of genes as sociated with differentiation programmes.

Long-term reconstitution Lifelong renewal of tissue by transplanted cells. The definitive assay for haematopoietic, epidermal and spermatogonial stem cells. Transplantation assay may not be appropriate for all tissues.
Niche Cellular microenvironment providing support and stimuli necessary to sustain self-renewal.

Plasticity Unproven notion that tissue stem cells may broaden potency in response to physiological demands or insults.

Potency The range of commitment options available to a cell. Totipotent Sufficient to form entire organism. Totipotency is seen in zygote and plant meristem cells; not demonstrated for any vertebrate stem cell.

Phuripotent Able to form all the body's cell lineages, includinggerm cells, and some or even all extraembryonic cell types. Example: embryonic stem cells.

Multipotent Can formmultiple lineages that constitute an entire tissue or tissues. Example: haematopoietic stem cells.

Oligopotent Able to form two or more lineages within a tissue. Example: a neural stem cell that can create a subset of neurons in the brain. Unip otent Forms a single lineage. Example: spermatogonial stem cells.

Progenitor cell Generic term for any dividing cell with the capacity to differentiate. Includes putative stem cells in which self-renewal has notyet been demonstrated.

Regenerativemedicine Reconstruction of diseased or injured tissue by activation of endogenous cells or by cell transplantation.

Reprogramming Increase in potency. Occurs naturally in regenerative organisms (dedifferentiation). Induced experimentally in mammalian cells by nuclear transfer, cell fusion, genetic manipulation or in vitro culture.

Self-renewal Cycles of division that repeatedly generate at least one daughter equivalent to the mother cell with latent capacity for differentiation. This is the defining property of stem cells.

Stem cell A cell that can continuously produce unaltered daughters and also has the ability to produce daughter cells that have different, more restricted properties.

Stem-cell homeostasis Persistence of tissuestem-cell pool throughout life. Requires balancing symmetric self-renewal with differentiative divisions at the population level, or sustained asymmetric self-renewal.

Stemness Unproven notion that differentstem cells are regulated by common genes and mechanisms.

Tissue stem cell Derived from, or resident in, a fetal or adult tissue, with potency limited to cells of that tissue. These cells sustain turnover and repair throughoutlife in some tissues.

Transit-amplifying cell Proliferative stem-cell progeny fated for differentiation. Initially may not be committed and may retain self-renewal. 